\title{
A Balanced Strategy for the FFBS Operator Integrating Dispatch Area, Route, and Depot Based on Multimodel Technologies
}

\author{
Qingfeng Zhou $\mathbb{D},^{1,2}$ Jun Zhou $\mathbb{D}^{1},{ }^{1}$ and Chun Janice Wong $\mathbb{D}^{3}$ \\ ${ }^{1}$ Shenzhen Urban Planning \&Land Resource Research Center, Shenzhen 518055, Guangdong, China \\ ${ }^{2}$ Shenzhen Key Laboratory of Urban Planning and Decision Making, Shenzhen 518055, Guangdong, China \\ ${ }^{3}$ Harbin Institute of Technology, Shenzhen 518055, Guangdong, China \\ Correspondence should be addressed to Chun Janice Wong; janicewong@hit.edu.cn
}

Received 21 December 2020; Revised 26 April 2021; Accepted 10 May 2021; Published 20 May 2021

Academic Editor: Erfan Hassannayebi

Copyright ( 2021 Qingfeng Zhou et al. This is an open access article distributed under the Creative Commons Attribution License, which permits unrestricted use, distribution, and reproduction in any medium, provided the original work is properly cited.

Bicycle scheduling is the essential strategy for balancing the demand for the public bicycle system (PBS). Existing literature pays more attention to bike scheduling models and their solutions, but seldom discusses the dispatch area and depot center. Reasonable dockless public bicycle dispatch area and optimal dockless bike dispatch depot location in the service area were discussed from the existing shared bicycle operation data in this paper. We proposed a feasible framework including bike trip network segmentation, mean-shift clustering based on the point position, VRP model, genetic algorithm, and TOPSIS evaluation method. The effectiveness and superiority of the division of the dispatch area are verified. The main evidence for this article is (1) although the cycling networks of bicycles are different at different times of the day, the results of community division are relatively stable and have great similarities. (2) The plan of the dispatch area has impacted on the operation efficiency of the PBS. For a scheduling area, the target value of the optimal scheduling strategy corresponding to different dispatch centers is obviously different. Therefore, the location of the dispatch center has a great impact on the quality of the scheduling strategy. The dispatch area determined by bike trip OD community detection has stable characteristics of scheduling costs. (3) This work is an attempt to combine big data and model technology to assist city management. We build a feasible framework to serve a balanced strategy for FFBS which can provide reasonable dispatch area, optimal dispatch depot location, dispatch truck's route length, load action, and time window. Our proposed framework provides new ideas for regional traffic dispatching for the traffic management department and FFBS operator, which has certain practical reference significance.

\section{Introduction}

Public bike system (PBS), also called a bicycle-sharing system (BSS), which was born in 1965 in Europe, has been developed for three generations [1]. The concept of the PBS/ BSS is simple: a user arrives at a station, takes a bike, uses it for a while, and then returns it to another station. It is economical, eco-friendly, and healthy, has ultralow carbon emissions, is more equitable, has increasingly received attention in the last decade, and has rapidly emerged in many cities all over the world [2]. Since 2016, a relatively new model of the PBS, known as the free-float bike-sharing (FFBS) system, has increasingly gained its popularity. The FFBS is based on the mobile app and GPS which eliminates stations and docks (also called dockless bike). Passengers can easily pick up and drop off the bike anywhere using their cell phone. This system is quite spread nowadays through enterprises as OFO and Mobike since early 2016 in China.

The FFBS is an innovative bike-sharing model. FFBS saves on start-up cost, in comparison to station-based bike sharing (SBBS), by avoiding the construction of expensive docking stations and kiosk machines. FFBS prevents bike theft and offers significant opportunities for smart management by tracking bikes in real time with built-in GPS. Despite the convenience and flexibility provided to users and their contribution to the sustainability of urban transportation, FFBS systems also face numerous challenges. For SBBS, the lack of resources is the major issue: a user can arrive at a station that has no bike available or wants to return her bike at a station with no empty spot. Like SBBS, 
the success of FFBS depends on the efficiency of its rebalancing operations to serve the maximal demand as possible. It is not easy to maintain an effective balance in the distribution of bikes. The relationship between the supply and demand of free-float bikes is more complicated because of no restrictions on the use time and location. Owing to the fluctuating and asymmetric demand for rides throughout the day, the spatial distribution of bikes is highly imbalanced. Some scholars have studied the mobility patterns and imbalance characteristics of FFBS by analyzing its historical trip [3-5]. Their studies provide insights to assist the system operator to make more informed decisions. There are many research studies on public bicycle balance strategies. A widely adopted rebalancing tactic is the operator-based approach, characterized by a fleet of trucks and staff dedicated to manually transferring bikes across different regions. The strategy generally includes three contents: (1) determining the scope of the rebalance area; (2) determining the location of the dispatch center; and (3) finding the optimal dispatch strategy. Among them, the research on optimal dispatching strategies is the most concerned issue for scholars, and the scheduling objectives and solving algorithms have obtained rich results. However, a reasonable dispatching area is necessary for effective dispatching work. For those bicycle stations included in a dispatch area, if most stations in the area need to drop off bicycles, the bikes on the dispatch vehicle may be insufficient and need to return to the dispatch center for loading; if most stations in the area need to pick up bicycles, then the dispatching vehicle cannot quickly and effectively load these bicycles away. The reasonable dispatch area should maintain a balanced relationship between pickup and drop-off bikes.

In this study, we mainly paid more attention to the dispatching range and the location of the dispatching center for the FFBS. This paper is the first to comprehensively consider the scope of the rebalance area, dispatching route, and dispatching centers to provide a balanced strategy for FFBS system operators. The framework involves the network community structure, vehicle balancing problem, and multiobjective decision-making as shown in Figure 1. Two issues are discussed from the existing shared bicycle operation data: (1) how to determine a reasonable dockless public bicycle dispatch area? (2) How to find an optimal dockless bike dispatch depot location in the service area? In the framework, first, a network was established based on real bike trip OD data. Second, the social network theory was used to analyze the trip network structure by the community detection algorithm. The subgroups of the trip network can be obtained. The number of bicycles flowing between subgroup grids is relatively stable at a certain time. Therefore, the results of subgroup grids can be considered as a scope of the rebalance area. Third, for the rebalance area (grids of a subgroup), each grid is considered a potential dispatch center. We built a vehicle route problem model with two optimal objectives: the minimal vehicle cost and the maximum grid rebalance rate. The genetic algorithm mixed with Technique for Order Preference by Similarity to an Ideal Solution (TOPSIS) was used to find the optimal rebalanced strategy, and each potential dispatch depot's best rebalanced strategy records the vehicle path, vehicle cost, and maximum grid rebalanced rate. Last, bringing together the best strategies of all potential dispatch centers in a rebalance area, TOPSIS was applied again to select the best dispatch center by considering vehicle costs and grid rebalancing rates. Our proposed framework provides new ideas for regional traffic dispatching for the traffic management department and FFBS operator, which has certain practical reference significance.

The rest part of this paper is organized as follows: Section 2 presents a literature review on community detection and rebalancing operations in bike-sharing systems. In Section 3, we described the community detection algorithm, the vehicle balance problem model, and the genetic algorithm used. In Section 4, we described the data of the OFO bicycle trip and the establishment of the OD network in Shenzhen. In Section 5, we analyzed community identification of the OFO bike trip network subgroup in Shenzhen and demonstrated the process of the framework to select the optimal dispatch center and its corresponding rebalanced strategy. Finally, Section 5 summarizes the results of this study and provides direction in future studies.

\section{Literature Review}

Sharing bike involved in many areas of research, and it is broadly based on two perspectives: user perspective and system perspective. In the remainder of this section, we mainly review the literature on community detection and rebalancing operations in the PBS.

2.1. Community in Networks. Newman and Girvan [6] gave widely accepted and used definitions: a community is a subgraph containing nodes which are more densely linked to each other than to the rest of the graph. A graph has a community structure if the number of links into any subgraph is higher than the number of links between those subgraphs. Very promising research on complex network theory attains the detection of communities [7]. A graph consists of edges and vertices such as $G=G(V, E)$. Community detection is the identification of $n c \geq 1$ communities in $G$ such that the vertices of a community form an overlay of $V . C=\left\{C_{1}, C_{2}, \ldots, G_{n c}\right\}$. If the intersection of the vertices of any two communities is empty, $C$ is called a disjoint community; otherwise, it is called an overlapping community. Traditionally, community detection in graphs aims at identifying the modules only based on the topology. The problem has a long tradition, and it has appeared in various forms in several disciplines. New advances also propose the study of detection of communities in weighted networks where not only the topology influences the shaping of clusters but also the weight of each link. Many authors have proposed methods and algorithms to detect communities in networks. The literature indicates three main methods: divisive algorithms, optimization methods, and spectral methods.

Community detection in transportation network research is mostly used to discover urban activity structures. 


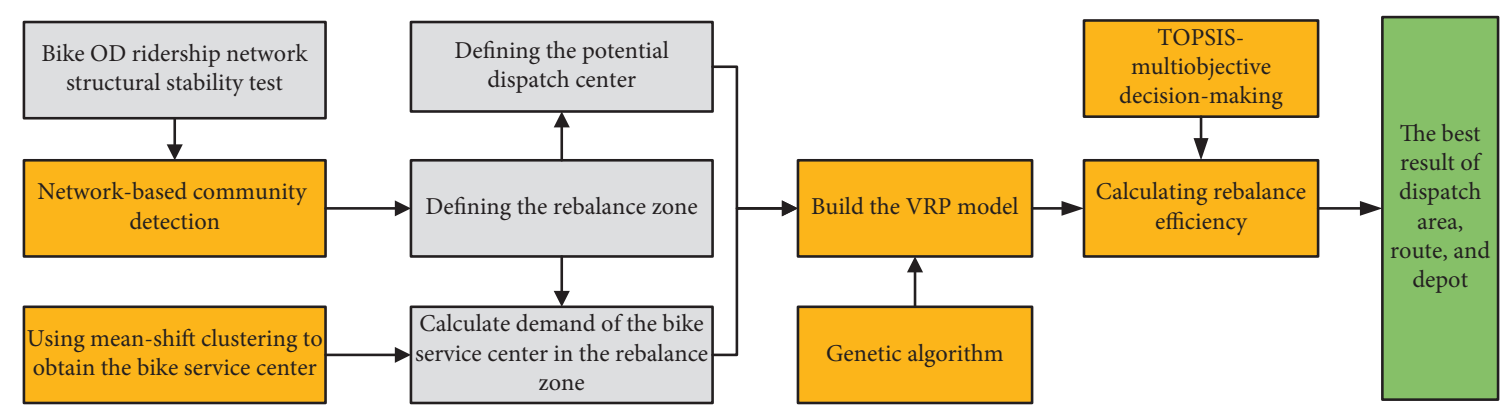

Figure 1: The workflow of this study.

De Montis et al. [8] adopted an algorithm based on the maximization of the weighted modularity of the commuter network to detect productive basins composed of municipalities. Du et al. [9] observed the dynamic mobility flows using community snapshots of different spatial stations over time using Shanghai as a case study. Zhang et al. [10] used community detection to evaluate the bus network topological structure. Austwick and Zaltz [11] employed community detection in the bicycle-sharing network to explore usage in cities. Borgnat et al. [12] discussed how Lyon's shared bicycle system, called Velo'v, is a dynamical complex network and how using community detection methods gives interesting results. Yao [13] constructed the public bicycle networks of different urban areas based on the real-time data of the Nanjing public bicycle system. Secondly, we analyzed and compared degree, strength, radiation distance, and community structure of the networks to understand the internal relations of the public bicycle system. In the next section, we illustrate the Louvain algorithm as proposed by Blondel et al. [14]. Therefore, we apply it to detect territorial clusters shaped by commuting in the center area of Shenzhen.

\subsection{Bike-Sharing Rebalancing Strategies. Bike rebalancing} strategies can be classified into user-based and operatorbased. User-based approaches promote customers to select more appropriate origins/destinations to realize a more balanced system. Operator-based approach emphasizes the optimal dispatching of trucks to manually transferring bikes across different areas which is a widely adopted rebalancing tactic.

2.2.1. The User-Based Strategies. Fare discounts or pricing schemes are the most used means to motivate users to change destinations for improving the operational performance of the PBS $[15,16]$. The optimized price vector determines the level of incentives that can persuade users to ride a bike from or stop at neighboring stations, thereby strategically reducing the number of unbalanced stations. Patel et al. [17] developed a discrete event simulation model of a real-world PBS to evaluate the effectiveness of incentives in rebalancing the system. Their job can consider profit and service levels to choose the best incentive plan. Reiss and Bosenberger [18] studied a user-based approach to a FFBS system and discussed the advantages and applicable scenarios for both operator-based and user-based relocation strategies. Wu et al. [19] employed user-based tactics by incentivizing users to perform repositioning activities and constructed a more detailed quantitative model which can derive the optimal incentive scheme for the FFBS system. Some studies considered both user-based and operatorbased strategies. Pfrommer et al. [20] used the model-based predictive control principle to examine the combination of dynamic truck routing and incentive scheme design in bicycle redistribution. Li and Shan [21] proposed two types of users in the BSS: leisure travelers and commuters. Operators and governments can adopt a bidirectional incentive model to improve their redistribution service levels. Ghosh and Varakantham [22] proposed a potentially self-sustaining and environment-friendly system of dynamic repositioning, which moves idle bikes during the day with the help of bike trailers. Their work can provide an optimization formulation that generates "repositioning" tasks.

2.2.2. The Operator-Based Strategies. The goal of the operator-based bike rebalancing problem is usually to find a minimum cost route for a vehicle and restore the inventory level at every bike station to its target value by picking up and delivering bicycles as necessary. The problems are classified into static bike rebalancing problem (SBRP) and dynamic bike rebalancing problem. The SBRP concerns the task of repositioning bikes among stations when traffic is low, and the PBS is idle [23]. The term static refers to the assumption that the number of bicycles at each station is known in advance and will not change during the pickup and transfer operations. The bike numbers cannot be adjusted in real time. Contrary to the static problem, for dynamic repositioning, the number of bicycles may change during the operations due to users renting and returning bicycles. Operator transfers and user trips occur simultaneously, so more real-time information must be considered in dynamic repositioning [24, 25]. There are various models and solution methods proposed to address the repositioning problem. Cruz [26] dealt with the SBRP of one single vehicle available, and the objective is to find a least-cost route that meets the demand of all stations and does not violate the vehicle capacity limits in the tour. An iterated local search-based heuristic was used to solve the problem. Based on the investigation of the net flow of each bike-sharing station in Jersey City, Zhang [27] proposed an integer linear 
programming formulation to model bike-sharing static rebalancing. The formulation considers the problem introduced by the need to collect bicycles in need of repair, and a hybrid discrete particle swarm optimization algorithm is used to solve the model. Tang [28] proposed a bilevel programming model to formulate the SBRP, which can be used to determine the number of bikes loaded and unloaded at each station and the optimal truck routes in bike-sharing systems. Chemla et al. [29] presented efficient algorithms to solve the SBRP. Similar studies are available in the literature [30-32]. The multiple-vehicle balancing problem (MVBP) has the same objective that requires to design a set of routes and pickup and delivery operations along each route with multiple vehicles available. Casazza et al. [33] dealt with the MVBP and proposed an integer linear programming formulation obtaining proven optimal solutions for MVBP instances with up to 25 stations and an unbounded number of vehicles. Ho and Wai [34] proposed a hybrid large neighborhood search for solving the MVBP. Their heuristic is evaluated on three sets of instances with up to 518 stations and five vehicles. Júnior [35] presented an integer programming formulation, implemented under a branchand-cut scheme, in addition to an iterated local search metaheuristic that employs efficient move evaluation procedures. Ghosh et al. [36] proposed an optimization formulation to reposition bikes using vehicles while also considering the routes for vehicles and future expected demand. They decomposed the problem (bike repositioning and vehicle routing) and aggregated bike stations to reduce the computation time significantly. Szeto and Shui [37] investigated the routes of the repositioning vehicles and the loading and unloading quantities at each bike station to firstly minimize the positive deviation from the tolerance of total demand dissatisfaction and then service time. This set of strategies is then embedded into an enhanced artificial bee colony algorithm to solve the BRP.

For the FFBS, the research on the bike balance problem is growing. Pal and $\mathrm{Yu}$ [38] presented a novel mixed-integer linear program for solving the SBRP in a series of studies of FFBS planning and management. The proposed formulation can not only handle single as well as multiple vehicles but also allows for multiple visits to a node by the same vehicle. They used a hybrid nested large neighborhood search with variable neighborhood descent algorithm, which is both effective and efficient in solving static complete rebalancing problems for large-scale bike-sharing programs. Aiming at the BRP scheduling with travel uncertainty, a multiobjective integer programming model was established based on the consideration of the static demand of fix time period, station capacity limit, penalty cost, and other practical factors by Zhang and Zhang [39]. An algorithm based on "ant colony algorithm" is then given to solve the model. Liu [40] studied the FFBS bike repositioning problem with multiple depots, multiple visits, and multiple heterogeneous vehicles. Easily and hardly access nodes with different penalties are defined to represent different convenience levels of getting bikes from the FFBS. The objective of the repositioning is to minimize the weighted sum of the inconvenience level of getting bikes from the system and the total unmet demand and the total operational time. To solve this problem, an enhanced version of chemical reaction optimization is developed.

From the previous review, the research on the bikesharing rebalancing problem can be distinguished from the objective of the balanced strategy and the algorithm for solving it. The dispatching area and dispatching center are rarely discussed. For the FFBS, since the dockless bicycle does not have a centralized station, the starting and ending position of the vehicle are only related to the user's personal travel destination. This may result in different characteristics of bicycles using and influencing factors from the PBS. Our work complements previous research. This paper comprehensively considers the scope of the rebalance area, dispatching route, and dispatching centers to provide a balanced strategy for FFBS system operators.

\section{Method and Data}

\subsection{Community Detection}

3.1.1. The Modularity. In this paper, we mainly used the Louvain algorithm proposed by Blondel et al. [14] to identify the community structure of the bike trip network. The Louvain algorithm is an optimization method based on the maximization of an objective function called modularity [6], defined as follows for the case of weighted networks:

$$
Q_{w}=\frac{1}{2 W} \times \sum_{i j}\left(w_{i j}-\frac{s_{i} s_{j}}{2 W}\right) \times \delta\left(c_{i}, c_{j}\right) .
$$

In equation (1), $W_{i j}$ is the weight of the edge connecting node $i$ and node $j . s_{i}=\sum_{j} w_{i j}$ (called node strength) is the sum of the weights of the edges attached to node $i . s_{j}=\sum_{i} w_{i j}$ is the sum of the weights of the edges attached to node $j$. $W=1 / 2 \sum_{i j} w_{i j}$ is the sum of all the edge weights. $\delta\left(c_{i}, c_{j}\right)$ is a function. When vertices $i$ and $j$ belong to the same community, $\delta\left(c_{i}, c_{j}\right)$ is equal to 1 ; otherwise, its value is 0 . The modularity is used to quantify how good is a community subdivision among all possible ones. For a particular subdivision, how many edges are there inside the communities with respect to the number of edges among them can be measured by computing modularity. Its values range from -1 to +1 . The 0 value occurs when a certain subdivision has no more intracommunity edges that one would expect by random chance. A negative value means that there is no advantage in splitting the network in communities, and the best solution is one community.

3.1.2. The Louvain Algorithm. The Louvain algorithm allows one to approach two critical issues of optimization methods: detecting communities in large networks in a short time and considering the hierarchical community structure. The algorithm is based on an iterative process and can be used for both weighted and unweighted networks. For a network, the steps to community detection using the Louvain algorithm are as follows:

Step 1: each node is assigned to a unique single community. 
Step 2: neighbor nodes of each target node are preferentially included in the same community if the variation of the modularity $\Delta Q_{w}$ is positive. The $\Delta Q_{w}$ function measures the level of performance of the partition associated to the displacement of a node from a community $C$ to another.

Step 3: this aggregation process proceeds until the modularity function $Q_{w}$ reaches a maximum.

Step 4: a new network is then built whose nodes correspond to the communities obtained in Step 3; each link connecting a pair of communities is featured by a weight equal to the sum of the weights of the external links originally between them. The internal links are represented by a self-loop, whose weight is equal to the sum of their internal weights.

Step 5: Step 1 applies to the last network.

3.1.3. The Adjusted Rand Index. In order to measure bike trip network community changes over time, this study introduces the Rand index. From a mathematical standpoint, Rand index is related to the accuracy, but is applicable even when class labels are not used. The Rand index is computed as the ratio of the number of pairs of objects having the same label relationship in two partitions. The Rand index has two shortcomings: it does not take a constant value for two random partitions and does not provide suitable results when the data comprise categories that overlap with each other to some degree. As the adjusted Rand index (ARI), Hubert and Arabie [41] extended the basic Rand index and provided a method able to handle two partitions $R$ and $Q$ of the same dataset. The adjusted Rand index is defined as the following equation:

$$
\omega_{a}=a-\frac{(a+c)(a+b) / d}{(a+c)+(a+b) / 2-(a+c)+(a+b) / d} .
$$

In equation (2), $a$ is the number of pairs of objects belonging to the same class in $R$ and to the same cluster in $Q$. $b$ is the number of pairs of data objects belonging to the same class in $R$ and to different clusters in $Q . c$ is the number of pairs of objects belonging to different classes in $R$ and to the same cluster in $Q$. $d$ is the number of pairs of objects belonging to different classes in $R$ and to different clusters in $Q$. The adjusted Rand index gives the degree of agreement between two partitions of a dataset by a value bounded above by 1 . A high adjusted Rand index indicates a high level of agreement, while a value of 1 suggests a perfect agreement. In the case of random partitions, the adjusted Rand index gives a value of 0 .

3.2. VRP Model. Vehicle routing problem (VRP) is generally defined as follows: for a series of loading points and unloading points, organize appropriate driving routes to pass them in an orderly manner, while meeting certain constraints (such as the demand for goods, the amount of delivery, and the time of delivery), vehicle load capacity, total route length, demand time window, etc., to achieve the goal of a certain problem (such as the shortest distance, the least cost, the least time, and the least number of vehicles). Because the demand of bicycles will change, this study establishes a multivehicle and multitime window VRP model based on considering the time demand and location demand of bicycles.

3.2.1. The Working Conditions and Boundaries of the VRP Model. We set some of our model working conditions and boundaries:

(1) The bicycle demand of the service center is updated every hour. In a time, if the service center needs to be rebalanced, the demand of this bike service center is unchanged during the dispatch process.

(2) For a truck, the start and end of its dispatch route are in the same depot.

(3) If the service center needs to be rebalanced, it has one and only one dispatch truck to serve.

(4) The service center's bike demand is complemented as much as possible by taking advantage of demand differences between service centers. Bicycle dispatch operation logic when the truck reaches grid $i$ can be expressed by the following equation:

$$
q_{i}= \begin{cases}\max \left(D_{i}, C_{i}-G_{\max }\right) & \text { when } D_{i}<0, \\ \min \left(D_{i}, C_{i}\right) & \text { when } D_{i}>0 .\end{cases}
$$

In equation (3), $q_{i}$ is the drop/collect demand of bicycles in service center $i$. $D_{i}$ represents the needs of service center $i$; $C_{i}$ is the bike number loaded by the truck when the truck arrives at service center $i$. $D_{i}>0$ means need to drop bikes. If bicycles loaded by the truck are more than the demand, then the drop number of bikes is $D_{i}$; otherwise, the dropped number is $C_{i}$. If $D_{i}<0$, the truck needs to collect the bikes and transfer them to other service centers as much as the truck capacity allows. $G_{\max }$ is the truck capacity of the bicycle.

(5) When the dispatch truck departs from the depot, the initial number of shared bicycles loaded is determined according to the drop/collect number of bicycles of the first service center $D_{1}$. If $D_{1}<0$, the initial number of shared bicycles loaded is 0 . And if $D_{1}>0$, the initial number is $\min \left(D_{1}, G_{\max }\right)$.

3.2.2. The Objectives of the VRP Model. The dispatch objectives in the VRP of this study mainly consider two aspects:

(a) Minimal total route length of all dispatch trucks

(b) Maximum demand satisfaction rate

The objective function is given in the following formula: 


$$
\left\{\begin{array}{l}
\min S_{1}=\sum_{k=1}^{m}\left(d_{0 i} S_{i k}+\sum_{j=1}^{n} \sum_{i=1}^{n} d_{i j} x_{i j k}+d_{j 0} E_{j k}\right) \\
\max S_{2}=\sum_{i=1}^{m}\left(\left|\frac{q_{i}}{D_{i}}\right|\right)
\end{array} .\right.
$$

$S_{1}$ is the route length of all dispatch trucks. $k$ is the $k$ th dispatch truck. $x_{i j k}=1$ or $0 . x_{i j k}$ represents whether truck $k$ is from service center $i$ to service center $j$. Its value is 1 or $0 . d_{i j}$ is the distance between service center $i$ and center $j . d_{0 i}$ is the distance from the depot to service center $i$. $S_{i k}$ represents whether service center $i$ is the first destination of truck $k . d_{j 0}$ is the distance from the depot to service center $j . E_{j k}$ represents whether service center $j$ is the last destination of truck $k . S_{2}$ is the ratio of drop/collect bike number to drop/collect demand.

The input variables and output variables of the model are shown in Table 1.

3.2.3. The Algorithm of Solving the VRP. Genetic algorithm is one of the commonly used methods to solve the VRP. In this study, an elite strategy was introduced into the genetic algorithm to directly add the best individuals from the previous generation to the next generation of the population. The evolution direction of the evolution operator is one way, that is, it only accepts the evolution of the scheduling cost in the lower direction.

Step 1: generate the initial population. Let the number of iterations $z=0$, use an integer arrangement to encode $N$ grids, and use the coding sequence as the grid access order.

Step 2: randomly generate a series of demand points, and then add each demand point to the current dispatch route in turn. The individual coding sequence in the population corresponds to a determined scheduling route. The NP sequence individuals of length $N$ are randomly generated to form the initial population.

Step 3: check whether the truck meets the conditions for returning to the depot center: (1) the truck capacity reaches full load, and the next service center needs to collect bikes; (2) the truck is empty, and the next service center needs to drop bikes; (3) the time when the truck arrives at the next center is not within the time window of the center that needs to serve.

If they are met, add the demand point to the next delivery route; if not, add it to the current delivery route as shown in Figure 2.

Step 4: calculate fitness values for all individuals in the $z$-generation population. Individuals to be evolved are selected through roulette. The greater the fitness, the greater the probability that individuals will be selected.

Step 5: let $z=z+1$; increase the maximum fitness of the $z$-generation to the $z+1$-generation population. Determine whether it has reached the number of iterations
$z=\mathrm{NG}$; if yes, output the current population, that is, the optimal population, and execute Step 6.

Step 6: find the scheduling cost $S 1$ and delivery rate $S 2$ of the routes corresponding to all individual sequences in the optimal population. According to the optional range of cost and delivery rate provided by the decision maker, a set of candidate routes $U$ that satisfy the decision limit is screened. Establish a feature matrix based on the route set and corresponding target values, and use TOPSIS to determine the optimal route.

Step 7: TOPSIS (Technique for Order Performance by Similarity to an Ideal Solution) is based on simultaneous minimization of distance from an ideal point (IP) and maximization of distance from a nadir point (NP), and the optimal route is selected according to the length and demand satisfaction rate of each route by using TOPSIS. Here, we omit the evaluation process of TOPSIS and directly list the evaluation results of the strategy.

3.3. Data. A network was established based on real bike trip $\mathrm{OD}$ data. The analysis was conducted employing trip data for the OFO bicycle-sharing system. We scanned the working status of these bicycles every 15 minutes in one week of September 2017. There are about 57.6 million bicycle status records in a day. For a bicycle ID, we first judge whether the bicycle is used by comparing whether its position has changed. If changed, we saved the time and position of the bicycle. Then, according to the average travel speed and a travel distance of the bicycle, the abnormal bicycle use record is rejected. Figure 3 is a summary of the number of times the shared bicycles are used in 24 hours. Based on the data presented in Figure 3, we extracted the four periods of the day (morning: 07:00-10:00; noon: 11:00-14:00; afternoon: 15:00-18:00; evening: 19:00-22:00).

First, since there are no fixed stations for the dockless bike, we refer to the methodology for assessing the impact of floating traffic [42]. The service area of FFBS is divided into grids. Because $71.25 \%$ of bicycle trips have more than 500 meters of trip distance, 500 meters is selected as the scale for dividing the grid of Shenzhen. Our final dataset comprises 220,042 unique bicycle trips distributed over 619 grids in Shenzhen (see Figure 4). Second, the bike trips were sorted in time sequences including trip ID, pickup time and location, and drop-off time and location. The involved location is merged into the grid closest to it. Third, aggregated on a grid level, our data can be used to compile an origin-destination (OD) matrix. In the last step, a spatial network is constructed from the OD matrix, taking grids as nodes, trips 
TABLE 1: The input variables and output variables of the VRP model.

\begin{tabular}{|c|c|c|}
\hline & e input & The output \\
\hline 1 & $\begin{array}{l}\text { Road network for calculating route length and the time spent on the } \\
\text { road }\end{array}$ & Optimal location of the depot \\
\hline 2 & Speed of the dispatch truck & Number of $\mathrm{t}$ \\
\hline 3 & Capacity of the truck & 3 Number of bikes loaded/collected at each servic \\
\hline 4 & Operation time of dropping/collecting bikes at the service center & Each truck's route length \\
\hline 5 & Locations of the potential depot & Total route length of all dispatch trucks \\
\hline 6 & Locations of the service center and their demands & Time schedule of the dispatch truck \\
\hline 7 & Time window of the service center needs to be rebalanced & Rebalance rate of all service centers \\
\hline
\end{tabular}

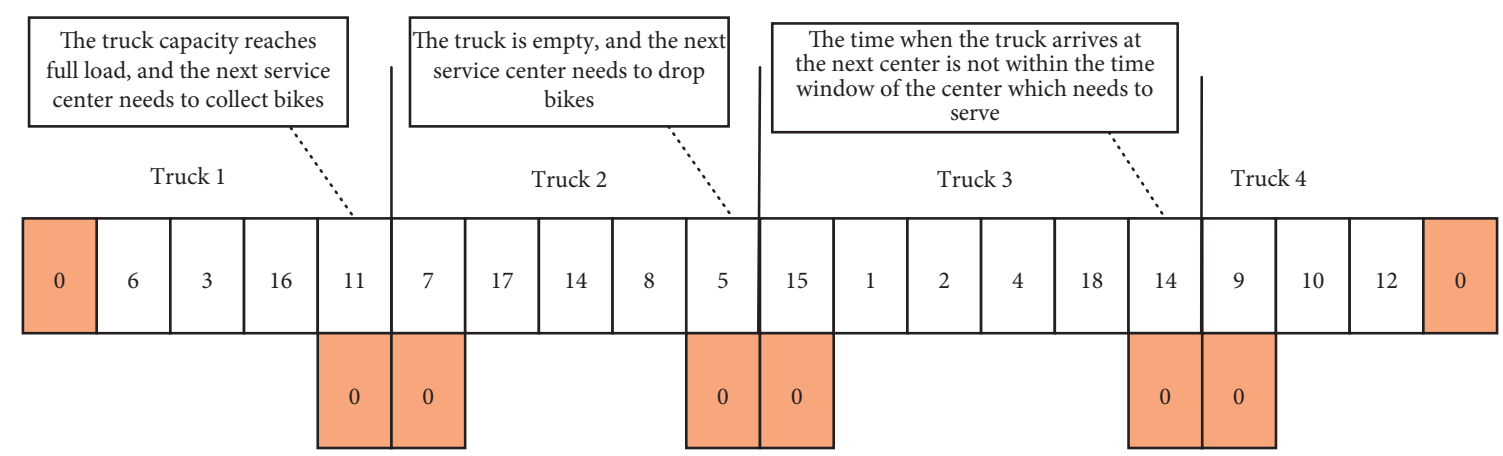

Figure 2: The conversion of demand point series into truck routes.

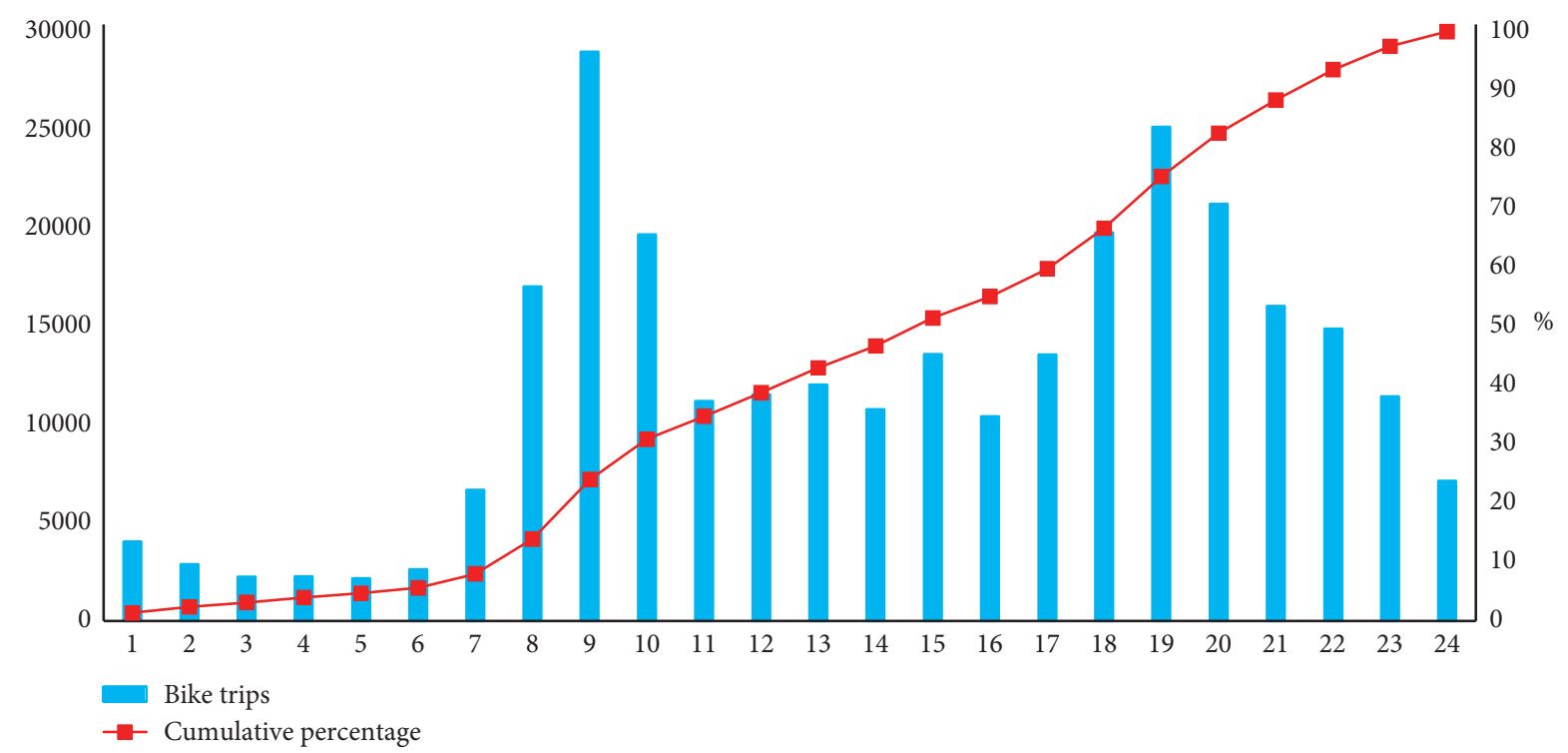

FIGURE 3: Floating bicycle riding trips' distribution across time within one day (24 hours).

between locations as edges, and the number of trips as the weight of edges. The nodes in the network are clustered according to the tightness of the connection, and the community division of the traffic network is obtained. We can then formulate a graph $G$ with each grid describing a network node $N$, linked to every other grid in the network by a set of directed edges $E$ weighted by a flow equal to the number of trips observed, given in the OD matrix.

\section{Results and Discussion}

4.1. The Structure and Stability of Bike OD Network Communities. In this section, we present an application of the community detection methodology described in Section 3 to support determining a reasonable dockless public bicycle dispatch area. Figure 5 shows the spatial distribution of community detection in four periods. In the figure, the same 


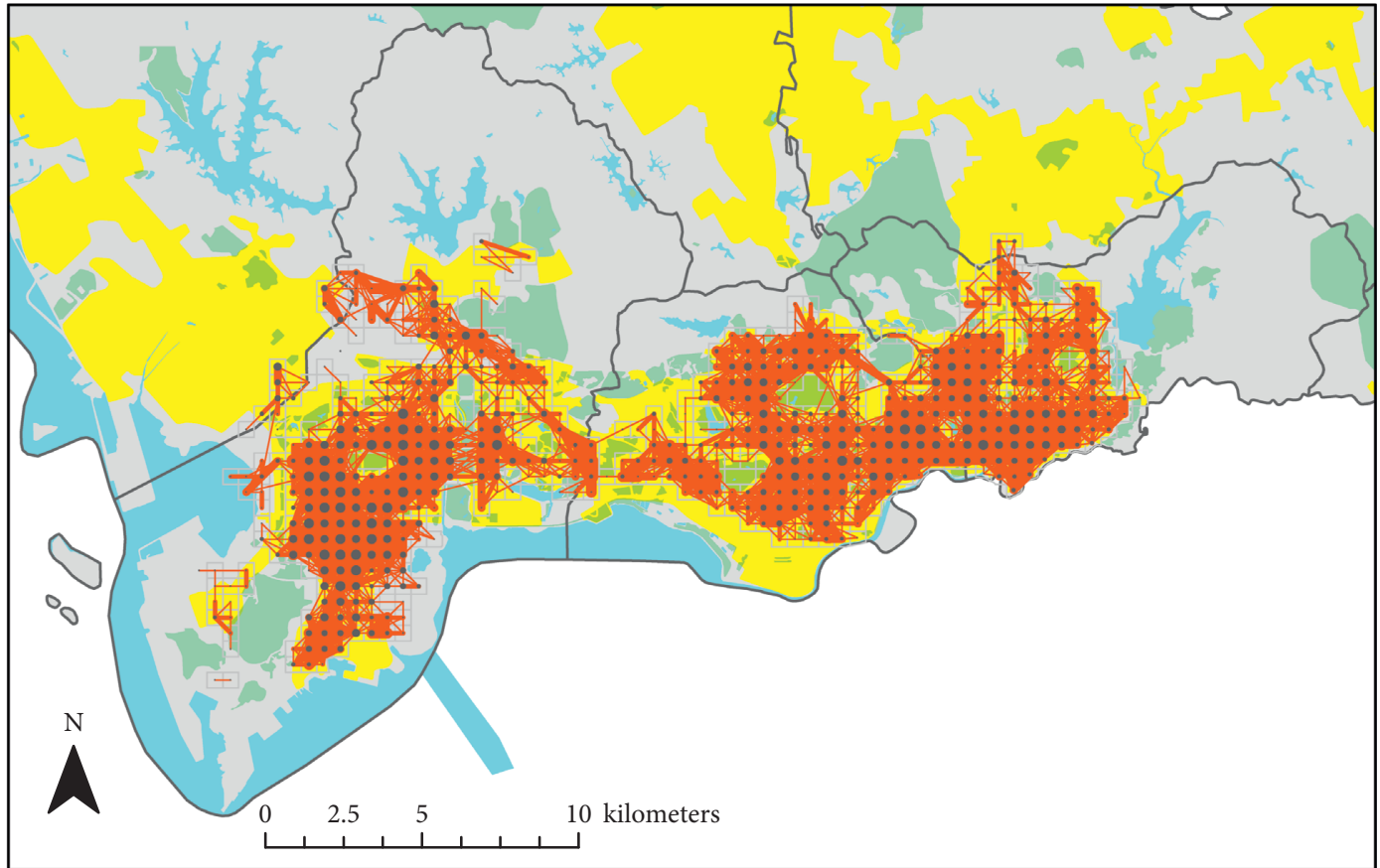

Grid_center_degree

$\begin{array}{ll}2-22 & -10-24 \\ 23-37 & -25-45 \\ 38-54 & -46-75 \\ 55-73 & 76-115 \\ 74-107 & 116-210\end{array}$

Figure 4: The OD network in Shenzhen.

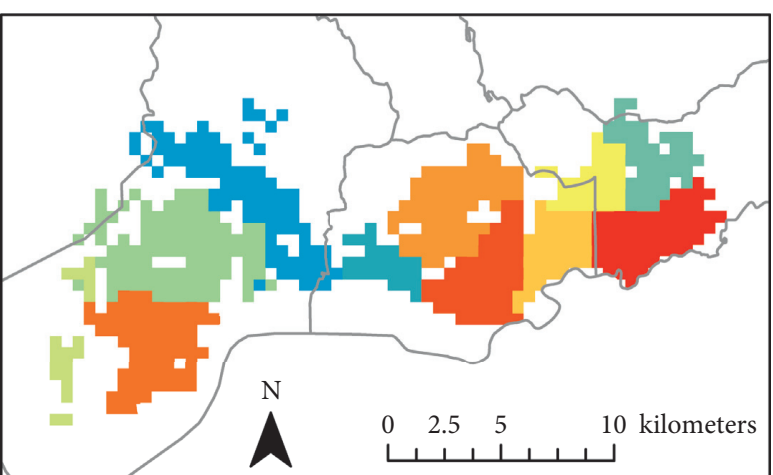

(a)

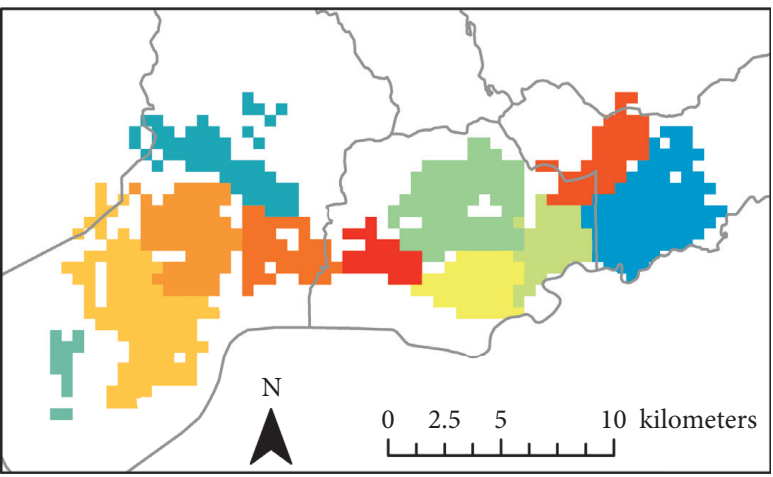

(c)

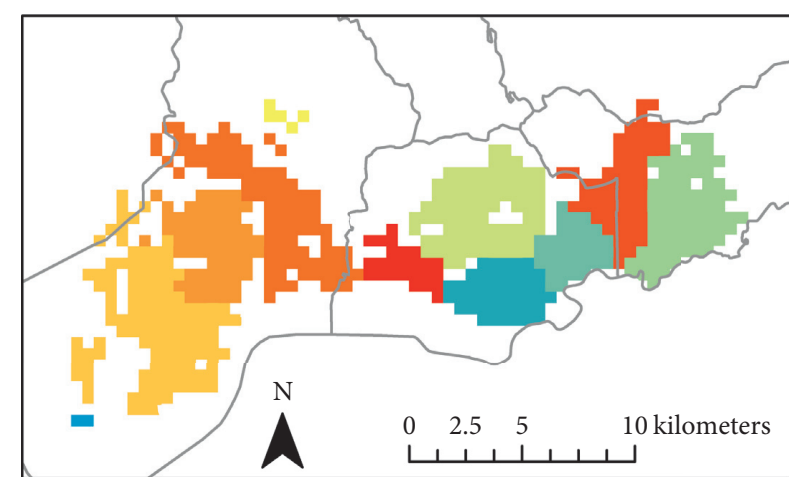

(b)

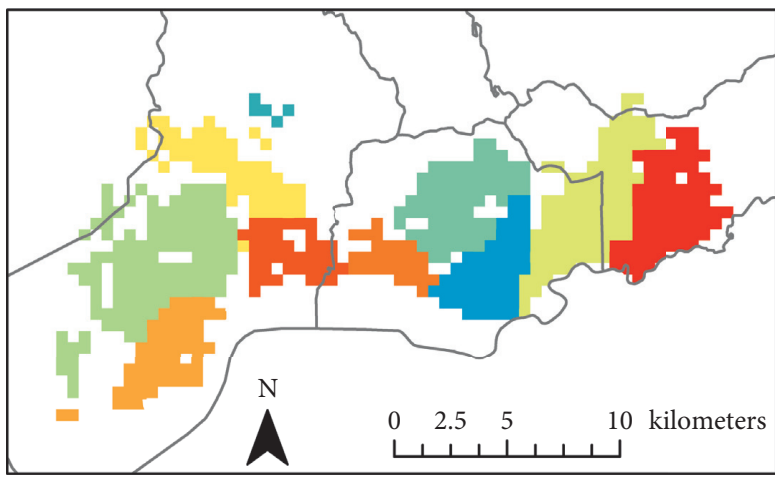

(d)

FiguRE 5: Spatial distribution of community detection in four periods. (a) Community detection in 07:00-10:00. (b) Community detection in 11:00-14:00. (c) Community detection in 15:00-18:00. (d) Community detection in 19:00-22:00. 
color grid indicates that it belongs to the same community. In the four time periods, the boundaries between most communities are very clear and obvious. These obvious boundaries are mainly due to the division of high-level roads and other natural terrains. In addition, the grids of a community are also spatially adjacent to each other, and it is rare for a community to contain other community grids. This is because public bikes are mainly for short-distance travel, and there are few long-distance cross-regional cycling records. A few small grids that are spatially isolated from most grids will form a small community. In Table 2, we summarize and compare the number of communities for four periods. For the modularity, the community modularity of the four periods mostly exceeds 0.7 except for the evening period. This shows that the OD network community structure is good. In the morning and evening peak hours, the average degree of nodes (the number of pickup and drop-off in the grid) exceeds 100, and the other two periods are around 70. Judging from the number of communities, the number of communities in the four periods is very close, which is between 9 and 11 .

In Table 3, the values of the adjusted Rand index for the case study are reported. The results in Table 4 show that the period 11:00-14:00 and the period 15:00-18:00 (values around 0.7951) show the best performance in the adjusted Rand index analysis. Partitions of the two periods have the highest similarities. The community similarity between evening peaks and afternoons is the lowest (values around 0.6073), which indicates that the difference of the bike spatial mobility pattern between evening peaks and afternoons is the largest during a day. In general, the communities in the four periods are compared in pairs, the ARI are all above 0.6, and the community structure is relatively stable. By the application of the Louvain method to the bike OD network of Shenzhen, we have shown that the mobility structure of the FFBS is obvious and stable. This high-quality and stable community division could suggest a bike dispatch area configuration of service area in a work day.

\subsection{The Optimal Dispatch Depot and Route in a Dispatch Area}

4.2.1. The Demo Dispatch Area. Based on the results in Section 5, a community of morning peak in Luohu was chosen as a dispatch area to analyze the optimal dispatch depot and route for the bike balance. The community contains a total of 28 grids; see Figure 5(a) for the location of the example community. Any two grids are connected, and the distance between the grids is the straight-line distance between their centroids. We used the mean-shift clustering method to identify the clustering area of bicycles based on the position of the bicycle by each hour in 07:00-10:00 [43]. When the bandwidth is 300 meters, a total of 78 bicycle gathering areas are obtained. Figure 6 shows the bicycle gathering area identified by mean-shift clustering. In Figure 6, each cluster has a center point, and the buffer analysis was proposed to obtain the range of the bicycle gathering area. 300 meters of buffers were established by ArcGIS based
TABLE 2: The number of communities for each period in the center Shenzhen bike network.

\begin{tabular}{lcccc}
\hline Time & $\begin{array}{c}\text { Community } \\
\text { number }\end{array}$ & Modularity & $\begin{array}{c}\text { Average } \\
\text { degree }\end{array}$ & Trips \\
\hline 07:00-10:00 & 11 & 0.717 & 102 & 63,665 \\
11:00-14:00 & 11 & 0.764 & 63 & 39,097 \\
15:00-18:00 & 11 & 0.744 & 80 & 49,830 \\
19:00-22: & 10 & 0.698 & 108 & 67,450 \\
00 & & & & \\
\hline
\end{tabular}

on all cluster center points, thus to calculate the balance status of bikes in the bicycle gathering area.

We calculate the number of arrivals and departures of bicycles in each cluster and set a threshold. In a period, when the difference between arrival and departure times is more than 20, the bicycle gathering area is considered to need rebalancing service. By setting the threshold, there are 23 bicycle gathering areas that need to be rebalanced, called demand center, as shown in Figure 7. Different colors represent the time window requirements for rebalancing. The white number is the grid ID and represents a potential dispatch center. Table 4 lists the coordinates, requirements, and time of each demand center. In the drop/collect bike demand column, the negative sign indicates that the bicycle needs to be added, and the positive sign indicates that the bicycle needs to be transferred.

\subsubsection{The Generation of the Optimal Strategy for the Dispatch} Area. The initial variables set are as follows: the speed of the truck is $30 \mathrm{~km} / \mathrm{h}$, and it can load up to 100 bicycles. It takes 12-15 minutes for the demand center to collect bicycles. The algorithm of the VRP is implemented using MATLAB, and the algorithm iterations are 200. Every generation of population has 100 individuals, and everyone represents a dispatch route. The evolution rate and mutation rate are 0.8 and 0.05 , respectively. The algorithm starts with taking grid ID 4182 in Figure 7 as a potential dispatch center to find the best dispatch route. The best dispatch route is selected by comparing objectives of the VRP model. Figure 8 is a process diagram of finding the optimal dispatching strategy when the dispatching depot is set on grid ID 4182. Figure 8(a) records the change in the minimum number of trucks in each generation. With the iteration of the algorithm, the vehicle number quickly decreased from 6 to 3. After 130 iterations, the minimum number of vehicles is stable at 3 . Figure 8 (b) records the change in minimum total route length in each generation. The convergence process is very fast, and finally, it is stable at about $33 \mathrm{~km}$. The demand satisfaction rate in Figure 8(c) is also stable at about $99 \%$. It is worth noting that dispatch routes corresponding to the three optimal objectives are not the same dispatch route, so TOPSIS is needed for choosing the best strategy. After TOPSIS comprehensive evaluation, when grid ID 4182 is used as the dispatch center, the best strategy result is 3 trucks and $35.8 \mathrm{~km}$ route length with $86.63 \%$ of demand satisfaction rate. 
TABLE 3: The adjusted Rand index for different periods.

\begin{tabular}{lcccc}
\hline ARI & $07: 00-10: 00$ & $11: 00-14: 00$ & $15: 00-18: 00$ & $18: 00-22: 00$ \\
\hline $07: 00-10: 00$ & - & 0.6659 & 0.6397 & 0.6129 \\
$11: 00-14: 00$ & 0.6659 & - & 0.7951 & 0.6358 \\
$15: 00-18: 00$ & 0.6397 & 0.7951 & - & 0.6073 \\
$18: 00-22: 00$ & 0.6129 & 0.6358 & 0.6073 & - \\
\hline
\end{tabular}

TABLe 4: The spatial coordinates and requirements of the demand center in the demo dispatch area.

\begin{tabular}{lcccc}
\hline Demand center & $X$ & $Y$ & Drop/collect demand & Time window \\
\hline 1 & 12701734.11 & 2578973.88 & -32 & $06: 00-07: 00$ \\
2 & 12701795.72 & 2578366.42 & -27 & $07: 00-08: 00$ \\
3 & 12701474.83 & 2579484.32 & 33 & $07: 00-08: 00$ \\
4 & 12701722.34 & 2578711.72 & -25 & $07: 00-08: 00$ \\
5 & 12702581.54 & 2579159.77 & 73 & $07: 00-08: 00$ \\
6 & 12703056.19 & 2579551.61 & -39 & $07: 00-08: 00$ \\
7 & 12702346.15 & 2578537.59 & 39 & $07: 00-08: 00$ \\
8 & 12702957.21 & 2578930.22 & -37 & $07: 00-08: 00$ \\
9 & 12700627.12 & 2579357.52 & -27 & $07: 00-08: 00$ \\
10 & 12702186.49 & 2578514.40 & 87 & $08: 00-09: 00$ \\
11 & 12701879.39 & 2578922.07 & -63 & $08: 00-09: 00$ \\
12 & 12701553.92 & 2579136.82 & 32 & $08: 00-09: 00$ \\
13 & 12702440.59 & 2578885.01 & -38 & $08: 00-09: 00$ \\
14 & 12701450.30 & 2579466.74 & 80 & $08: 00-09: 00$ \\
15 & 12701073.24 & 2578938.58 & -74 & $08: 00-09: 00$ \\
16 & 12702980.11 & 2579419.16 & -48 & $08: 00-09: 00$ \\
17 & 12702978.05 & 2579848.00 & -40 & $08: 00-09: 00$ \\
18 & 12702416.17 & 2579948.83 & -25 & $08: 00-09: 00$ \\
19 & 12701963.87 & 2579839.53 & -56 & $08: 00-09: 00$ \\
20 & 12702922.36 & 2578921.61 & 59 & $08: 00-09: 00$ \\
21 & 12702473.52 & 2578904.86 & 43 & $09: 00-10: 00$ \\
22 & 12702413.21 & 2578535.34 & -82 & $09: 00-10: 00$ \\
23 & 12702971.81 & 2579505.02 & & $09: 00-10: 00$ \\
\hline & & &
\end{tabular}

After the above calculation process for each potential dispatch center in Figure 7, finally, we obtained 28 records of the best strategy. It can be considered that there are 28 dispatch center options, and the optimal dispatch center is evaluated based on the truck number, route length, and demand satisfaction rate corresponding to the dispatch center. Here, the TOPSIS method is used again. Table 5 records the corresponding values of the top five dispatch centers and the last five dispatch centers. We can find that the best dispatch center is grid ID 913. The target value of the optimal scheduling strategy corresponding to different dispatch centers is obviously different. Therefore, the location of the dispatch center has a great impact on the quality of the scheduling strategy.

Figure 9 is the optimal scheduling route for demo dispatch area 0 represents the dispatch center which is grid ID 913, and other numbers represent demand centers. The optimal scheduling strategy requires three trucks. The routes of the truck and details of operation and time at the demand center are shown in Table 6. The departure time of the three trucks from the dispatch center and the number of bicycles loaded are not the same. The time window of the demand center is all satisfied, and the demand satisfaction rate of each demand center is counted.
4.3. The Validation of Optimal Dispatch Divisions. Luohu District was used to verify the effectiveness of using the trip network community detection to determine the dispatch area. In the morning peak hours, the bicycle trip network of Luohu is divided into four communities according to the best modularity as shown in Figure 10(a). So, we use manual grouping (Figures 10(b) and 10(c)) to generate four comparison groups. Grids with same color indicate that they belong to the same scheduling area. Manual grouping ensures that each dispatch area is about the same size with a clear boundary, and no dispatch area is contained by another scheduling area.

Table 7 shows the scheduling cost of different division groups of dispatch area in Luohu District. First, for demand satisfaction rate, the total demand satisfaction rate of three division methods was not much different with all exceeding 90\%. The largest demand satisfaction rate is the result obtained by $\mathrm{M} 2$, which is close to $93 \%$, but the gap with the average demand satisfaction rate of $\mathrm{CD}$ is only $2 \%$. Second, for the VRP route length, the total dispatch route length of $\mathrm{CD}$ is the shortest, about $233.23 \mathrm{~km}$. The total dispatch route length of M1 and M2 is about $250 \mathrm{~km}$, at least $15 \mathrm{~km}$ more than CD. Third, for the dispatch truck number, CD needs 18 dispatch trucks, 

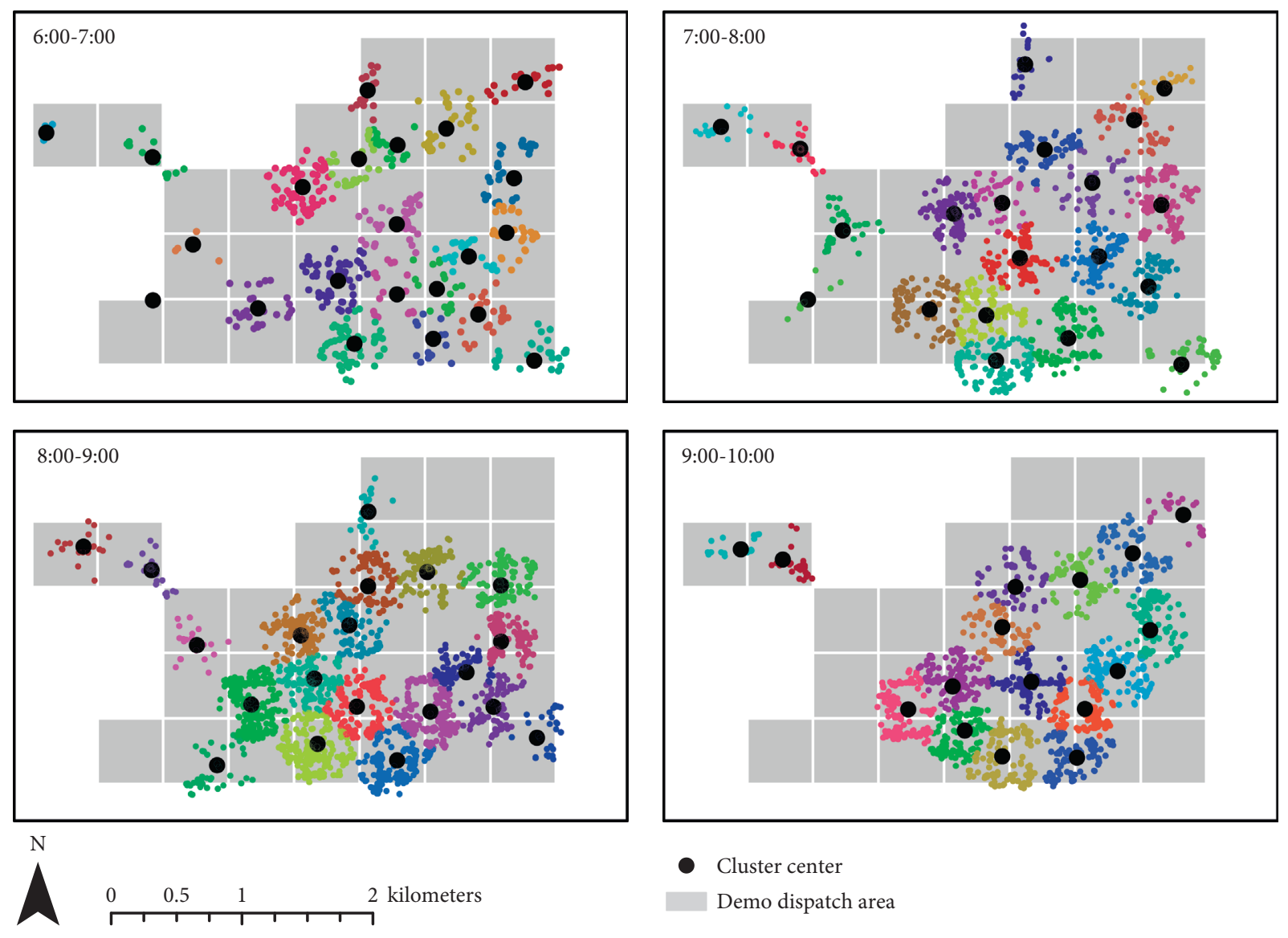

- Cluster center

Demo dispatch area

FIGURE 6: The bicycle gathering area identified by mean-shift clustering in four hours.

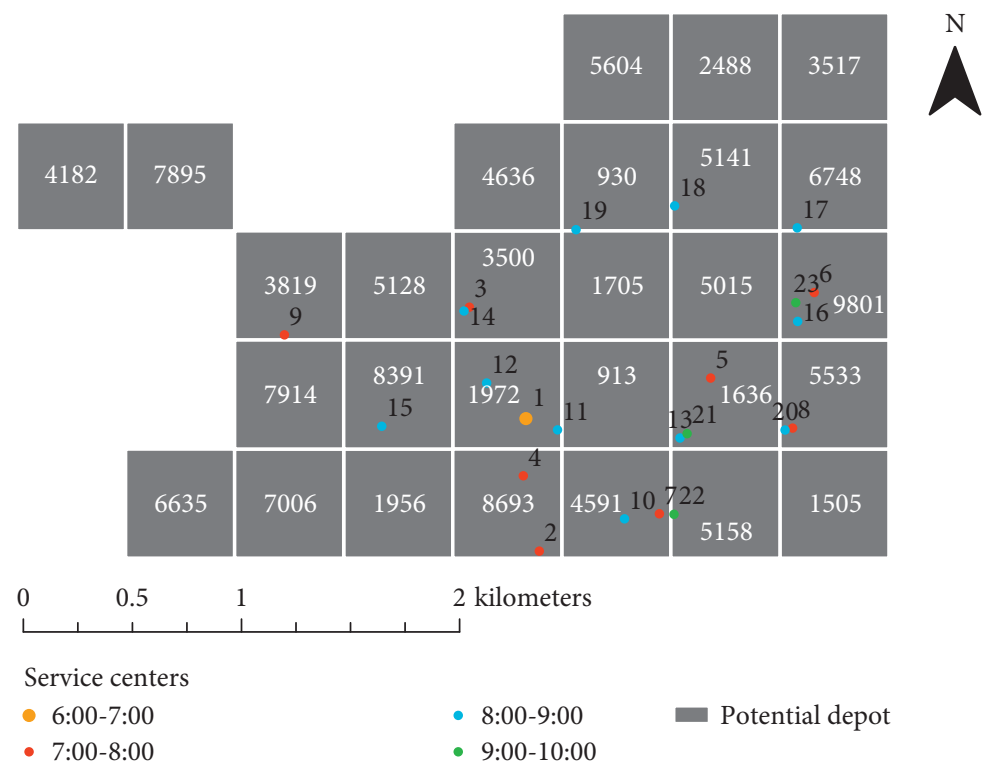

Figure 7: The demo dispatch area information in Futian.

while M1 and M2 need 19. In other words, in order to achieve the same demand satisfaction rate of the community detection group, manual group 1 and manual group 2 need to pay a lot of cost in the dispatch distance and truck. By comparing the results of scheduling strategies in different situations, we found that the 


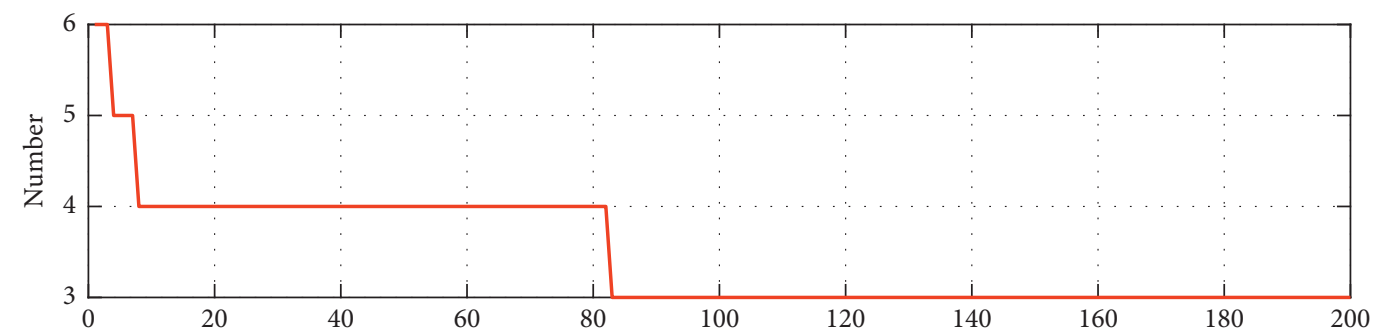

(a)

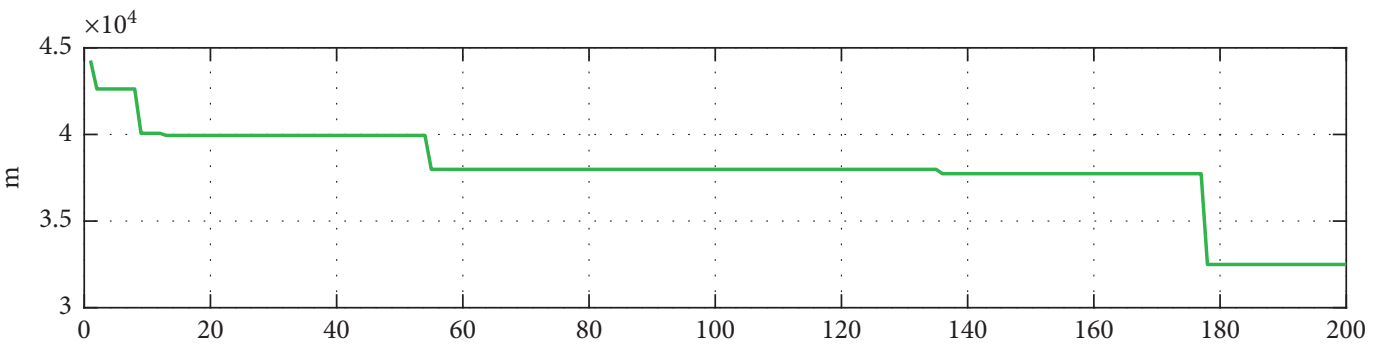

(b)

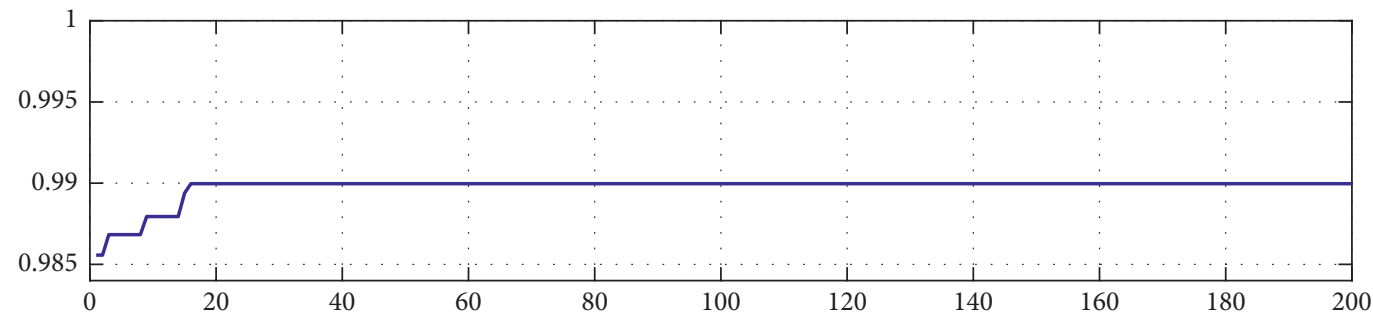

(c)

Figure 8: Process of seeking the optimal strategy. (a) Truck number. (b) Total length. (c) Demand satisfaction rate.

TABLE 5: The corresponding values of the top 5 and the last 5 dispatch centers.

\begin{tabular}{cccccc}
\hline Order & & Depot grid ID & Truck number & Route length $(\mathrm{km})$ & Demand satisfaction rate $(\%)$ \\
\hline \multirow{4}{*}{ Top 5 } & 1 & 913 & 3 & 20.80 & 91.04 \\
& 2 & 5128 & 3 & 26.81 & 92.52 \\
& 3 & 4182 & 3 & 35.99 & 90.15 \\
& 4 & 1972 & 3 & 26.18 & 90.10 \\
\hline \multirow{2}{*}{ Last 5 } & 5 & 9801 & 3 & 25.27 & 90.09 \\
& 5 & 3819 & 4 & 30.91 & 88.86 \\
& 4 & 4591 & 4 & 24.22 & 88.43 \\
& 3 & 5141 & 4 & 32.09 & 86.82 \\
& 2 & 1705 & 4 & 23.61 & 85.48 \\
\end{tabular}




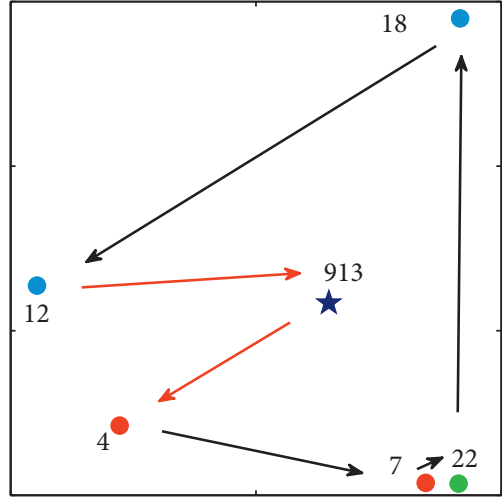

(a)

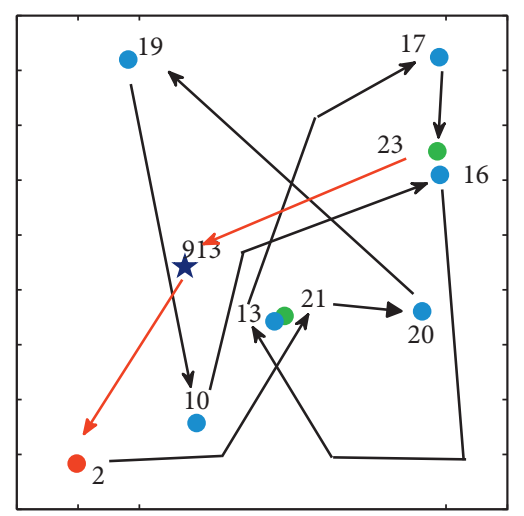

(b)

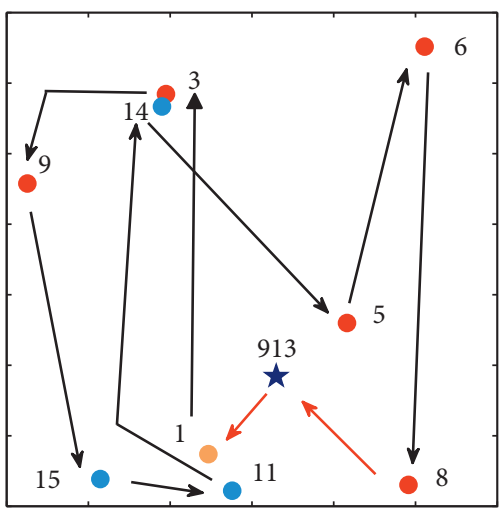

(c)

Figure 9: The optimal scheduling route of dispatch center 913. (a) Truck 1 route. (b) Truck 2 route. (c) Truck 3 route.

TABLE 6: The routes of the truck and details of operation and time at the demand center.

\begin{tabular}{|c|c|c|c|c|c|c|}
\hline Truck & Route & Arrive/leave & Time window & Drop/collect bike number & Drop/collect demand & Demand satisfaction \\
\hline \multirow{6}{*}{1} & 0 & - & - & 25 & & - \\
\hline & 4 & $6: 58 / 7: 13$ & 07:00-08:00 & -25 & -25 & $100 \%$ \\
\hline & 7 & $7: 18 / 7: 33$ & 07:00-08:00 & 39 & 39 & $100 \%$ \\
\hline & 22 & $7: 38 / 7: 53$ & 09:00-10:00 & 43 & 43 & $100 \%$ \\
\hline & 18 & $8: 00 / 8: 15$ & 08:00-09:00 & -40 & -40 & $100 \%$ \\
\hline & 12 & $8: 20 / 8: 35$ & 08:00-09:00 & 32 & 32 & $100 \%$ \\
\hline \multirow{10}{*}{2} & 0 & - & - & 27 & - & - \\
\hline & 2 & $6: 58 / 7: 12$ & 07:00-08:00 & -27 & -27 & $100 \%$ \\
\hline & 21 & $7: 13 / 7: 25$ & 09:00-10:00 & 59 & 59 & $100 \%$ \\
\hline & 20 & $7: 26 / 7: 38$ & 08:00-09:00 & -56 & -56 & $100 \%$ \\
\hline & 19 & $7: 40 / 7: 52$ & 08:00-09:00 & -3 & -27 & $11.10 \%$ \\
\hline & 10 & $7: 55 / 8: 09$ & 08:00-09:00 & 87 & 87 & $100 \%$ \\
\hline & 16 & $8: 10 / 8: 22$ & 08:00-09:00 & -74 & -74 & $100 \%$ \\
\hline & 13 & $8: 24 / 8: 36$ & 08:00-09:00 & 87 & 130 & $66.92 \%$ \\
\hline & 17 & $8: 38 / 8: 50$ & 08:00-09:00 & -48 & -48 & $100 \%$ \\
\hline & 23 & 8:52/9:04 & 09:00-10:00 & -52 & -82 & $63.41 \%$ \\
\hline \multirow{10}{*}{3} & 0 & - & - & 32 & - & - \\
\hline & 1 & $5: 59 / 6: 11$ & 06:00-07:00 & -32 & -32 & $100 \%$ \\
\hline & 3 & $6: 12 / 6: 24$ & 07:00-08:00 & 33 & 33 & $100 \%$ \\
\hline & 9 & $6: 26 / 6: 38$ & 07:00-08:00 & -27 & -27 & $100 \%$ \\
\hline & 15 & $6: 39 / 6: 51$ & 08:00-09:00 & 80 & 80 & $100 \%$ \\
\hline & 11 & $6: 53 / 7: 05$ & 08:00-09:00 & -63 & -63 & $100 \%$ \\
\hline & 14 & $7: 06 / 7: 18$ & 08:00-09:00 & -23 & -38 & $60.52 \%$ \\
\hline & 5 & $7: 21 / 7: 33$ & 07:00-08:00 & 73 & 73 & $100 \%$ \\
\hline & 6 & $7: 34 / 7: 46$ & 07:00-08:00 & -39 & -39 & $100 \%$ \\
\hline & 8 & $7: 47 / 7: 59$ & 07:00-08:00 & -34 & -37 & $91.89 \%$ \\
\hline \multicolumn{2}{|c|}{ Total length } & \multicolumn{2}{|c|}{$20.8 \mathrm{~km}$} & \multicolumn{2}{|c|}{ Demand satisfaction rate } & $91.04 \%$ \\
\hline
\end{tabular}




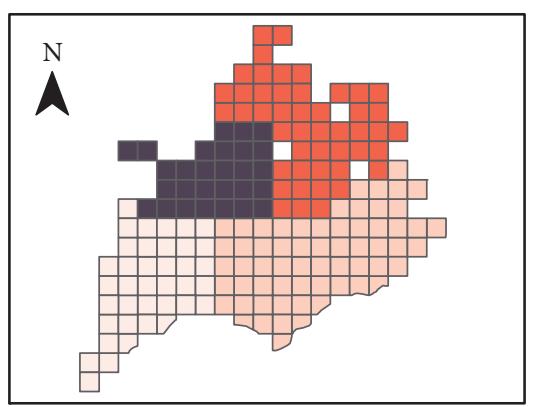

Sub dispatch area

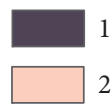

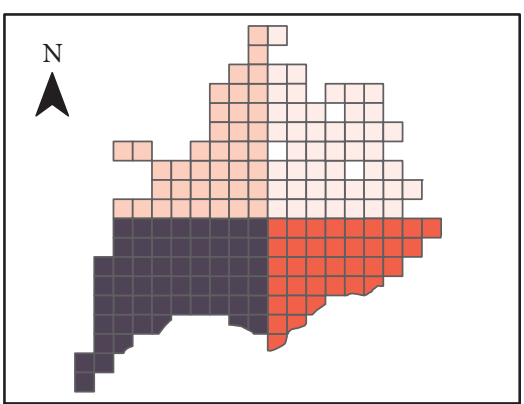

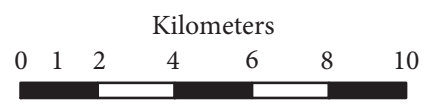

(b)

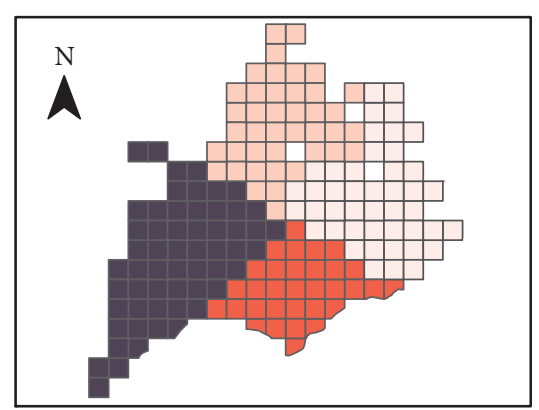

CD-community detection M1-manual group 1 M2-manual group 2

(c)

FIGURE 10: Three division groups of the dispatch area in Luohu District at morning peak.

TABle 7: The scheduling cost of different division groups of the dispatch area in Luohu District.

\begin{tabular}{|c|c|c|c|c|c|}
\hline Dispatch division & Subdispatch area & Depot grid & Truck number & Route length $(\mathrm{km})$ & Demand satisfaction (\%) \\
\hline \multirow{5}{*}{$\mathrm{CD}$} & 1 & 913 & 3 & 20.80 & 91.04 \\
\hline & 2 & 3128 & 7 & 109.92 & 89.80 \\
\hline & 3 & 6221 & 6 & 64.01 & 86.37 \\
\hline & 4 & 9764 & 2 & 38.50 & 93.99 \\
\hline & \multicolumn{2}{|c|}{ Total } & 18 & 233.23 & 90.3 \\
\hline \multirow{5}{*}{ M1 } & 1 & 6122 & 9 & 119.85 & 87.27 \\
\hline & 2 & 1972 & 4 & 48.71 & 85.91 \\
\hline & 3 & 8237 & 2 & 30.21 & 96.77 \\
\hline & 4 & 223 & 4 & 60.09 & 95.62 \\
\hline & \multicolumn{2}{|c|}{ Total } & 19 & 258.87 & 91.39 \\
\hline \multirow{5}{*}{ M2 } & 1 & 5817 & 9 & 113.64 & 88.58 \\
\hline & 2 & 930 & 4 & 57.00 & 92.77 \\
\hline & 3 & 5307 & 2 & 28.75 & 95.93 \\
\hline & 4 & 7356 & 4 & 49.81 & 93.56 \\
\hline & \multicolumn{2}{|c|}{ Total } & 19 & 249.20 & 92.71 \\
\hline
\end{tabular}

CD: community detection; M1: manual group 1; M2: manual group 2.

dispatch area determined by bike trip OD community detection has stable characteristics of scheduling costs. In general, the scheduling strategy found through the trip OD community detection is optimal. This shows that the division of the scheduling area is a very important issue, which has a great impact on the dispatch cost. The method proposed in this paper can provide a reference for dividing the scheduling area.

\section{Conclusions}

This paper establishes a network based on the bike trip data and then uses a community discovery algorithm to segment the cycling network. The vehicle routing problem model with the shortest dispatch route and satisfaction rate dual goals is established and solved, and the effectiveness and superiority of the division of the dispatch area are verified. The main evidence for this article is as follows:
(1) Many studies have shown that the bicycle riding has tidal characteristics, but our research found that the results of the community division of bicycle networks for different dispatches in a day are very similar, indicating that the flow of dockless shared bicycles is very stable and its range of activities has a clear boundary. From the perspective of the spatial characteristics of shared bicycle networks, the results of community division rarely show cross-regional phenomena. The interior of a community space will not contain another community, or a community will not be spatially separated by other communities. This provides the necessary basis for the division of bicycle dispatching areas.

(2) The plan of the dispatch area has impacted on the operation efficiency of the PBS. Our research shows that, for a scheduling area, the target value of the optimal scheduling strategy corresponding to different dispatch centers is obviously different. 
Therefore, the location of the dispatch center has a great impact on the quality of the scheduling strategy. By comparing the results of scheduling strategies in different situations, the dispatch area determined by bike trip OD community detection has stable characteristics of scheduling costs. In general, the scheduling strategy found through the trip OD community detection is optimal. The division of the scheduling area is a very important issue, which has a great impact on the dispatch cost.

(3) This work is an attempt to combine big data and model technology to assist city management. We build a feasible framework to serve a balanced strategy for FFBS integrating dispatching area, route, and depot. This framework includes the construction of the bike trip network and subnet segmentation, mean-shift clustering based on the point position, VRP model, genetic algorithm, and TOPSIS evaluation method, which can provide reasonable dispatch area, optimal dispatch depot location, dispatch truck's route length, load action, and time window. Our work provides new ideas for regional traffic dispatching for the traffic management department and FFBS operator, which has certain practical reference significance.

Our study also has some limitations. First, the working day operational data used only contain one week, so the results of the analysis may be biased. The data we analyzed did not include data for nonworking days. Second, in the real world, there are many restrictions on decision-making. Although the VRP model we adopt was considering time, truck capacity, and driving speed, it cannot handle complex situations such as multiple dispatch centers, real-time road condition information, and more detailed dispatch operation time. Third, in our proposed framework, the result of subnet segmentation is crucial to the generation of scheduling strategies. The construction of cycling networks at different times will affect the division of communities, and further exploration is needed in the future.

\section{Data Availability}

The bike data were supplied by authors of this article. They are freely available. Requests for access to these data should be made to Qingfeng Zhou (zhouqingfeng@hit.edu.cn).

\section{Conflicts of Interest}

The authors declare that they have no conflicts of interest.

\section{References}

[1] P. DeMaio, "Bike-sharing: history, impacts, models of provision, and future," Journal of Public Transportation, vol. 12, no. 4 , p. 3, 2009.

[2] S. A. Shaheen, S. Guzman, and H. Zhang, "Bikesharing in Europe, the americas, and asia," Transportation Research Record: Journal of the Transportation Research Board, vol. 2143, no. 1, pp. 159-167, 2010.
[3] J. Dong, B. Chen, L. He et al., "A spatio-temporal flow model of urban dockless shared bikes based on points of interest clustering," ISPRS International Journal of Geo-Information, vol. 8, no. 8, p. 345, 2019.

[4] X. Li, Y. Zhang, L. Sun, and Q. Liu, "Free-floating bike sharing in jiangsu: users' behaviors and influencing factors," Energies, vol. 11, no. 7, p. 1664, 2018.

[5] Y. Xu, D. Chen, X. Zhang et al., "Unravel the landscape and pulses of cycling activities from a dockless bike-sharing system," Computers, Environment and Urban Systems, vol. 75, pp. 184-203, 2019.

[6] M. Girvan and M. E. J. Newman, "Community structure in social and biological networks," Proceedings of the National Academy of Sciences of the United States of America, vol. 99, no. 12, p. 782, 2002.

[7] S. Fortunato, "Community detection in graphs," Physics Reports, vol. 486, pp. 75-174, 2009.

[8] A. De Montis, S. Caschili, and A. Chessa, "Commuter networks and community detection: a method for planning sub regional areas," The European Physical Journal Special Topics, vol. 215, no. 1, pp. 75-91, 2013.

[9] Z. Du, B. Yang, and J. Liu, "Understanding the spatial and temporal activity patterns of subway mobility flows," 2017, https://arxiv.org/abs/1702.02456.

[10] H. Zhang, P. Zhao, Y. Wang, X. Yao, and C. Zhuge, "Evaluation of bus networks in China: from topology and transfer perspectives," Discrete Dynamics in Nature \& Society, vol. 2015, Article ID 328320, 8 pages, 2015.

[11] Austwick and M. Zaltz, "The structure of spatial networks and communities in bicycle sharing systems," PLoS One, vol. 8, Article ID e74685, 2013.

[12] P. Borgnat, C. Robardet, P. Abry, P. Flandrin, JB. Rouquier, and N. Tremblay, “A dynamical network view of Lyon's vélo’v shared bicycle system," in Dynamics on and of Complex Networks, Volume 2. Modeling and Simulation in Science, Engineering and Technology, A. Mukherjee, M. Choudhury, F. Peruani, N. Ganguly, and B. Mitra, Eds., Birkhäuser, New York, NY, USA, 2013.

[13] Y. Yao, "Analysis of network structure of urban bike-sharing system: a case study based on real-time data of a public bicycle system," Sustainability, vol. 11, no. 19, p. 5425, 2019.

[14] V. D. Blondel, J. Guillaume, R. Lambiotte, and E. Lefebvre, "Fast unfolding of communites in large networks," Journal of Statistical Mechanics: Theory and Experiment, vol. 10, pp. 10008-10020, 2008.

[15] A. Febbraro, N. Sacco, and M. Saeednia, "One-way carsharing: solving the relocation problem," Transportation Research Record: Journal of the Transportation Research Board, vol. 2319, pp. 113-120, 2012.

[16] Z. Haider, "Inventory rebalancing through pricing in public bike sharing systems," European Journal of Operational Research, vol. 270, no. 1, pp. 103-117, 2018.

[17] S. J. Patel, R. Qiu, and A. Negahban, "Incentive-based rebalancing of bike-sharing systems," in Advances in Service Science, pp. 21-30, Springer, Berlin, Germany, 2018.

[18] S. Reiss and K. Bogenberger, "A relocation strategy for munich's bike sharing system: combining an operator-based and a user-based scheme," Transportation Research Procedia, vol. 22, pp. 105-114, 2017.

[19] R. Wu, S. Liu, and Z. Shi, "Customer incentive rebalancing plan in free-float bike-sharing system with limited information," Sustainability, vol. 11, no. 11, p. 3088, 2019.

[20] J. Pfrommer, J. Warrington, G. Schildbach, and M. Morari, "Dynamic vehicle redistribution and online price incentives in 
shared mobility systems," IEEE Transactions on Intelligent Transportation Systems, vol. 15, no. 4, pp. 1567-1578, 2014.

[21] L. Li and M. Shan, "Bidirectional incentive model for bicycle redistribution of a bicycle sharing system during rush hour," Sustainability, vol. 8, no. 12, p. 1299, 2016.

[22] S. Ghosh and P. Varakantham, "Incentivising the use of bike trailers for dynamic repositioning in bike sharing systems," in Proceedings of the International Conference on Automated Planning and Scheduling (ICAPS), Guangzhou, China, August 2017.

[23] M. Benchimol, A. Benchimol, F. Chappert et al., "Balancing the stations of a self service "bike hire" system," RAIRO-Operations Research, vol. 45, no. 1, pp. 37-61, 2011.

[24] C. Contardo, C. Morency, and L.-M. Rousseau, "Balancing a dynamic public bikesharing system," CIRRELT Technical Report, Centre Interuniversitaire de Recherche sur les Réseaux d'Entreprise, la Logistique et le Transport (CIRRELT), Montreal, Canada, 2012.

[25] C. Kloim, P. Papazek, B. Hu, and R. Gunther, "Balancing bicycle sharing systems: an approach for the dynamic case," in Evolutionary Computation in Combinatorial Optimisation, pp. 73-84, Springer, Berlin, Germany, 2014.

[26] F. Cruz, "A heuristic algorithm for a single vehicle static bike sharing rebalancing problem," Computers \& Operations Research, vol. 79, pp. 19-33, 2016.

[27] S. Zhang, "Bike-sharing static rebalancing by considering the collection of bicycles in need of repair," Journal of Advanced Transportation, vol. 2018, Article ID 8086378, 18 pages, 2018.

[28] Q. Tang, "A bilevel programming model and algorithm for the static bike repositioning problem," Journal of Advanced Transportation, vol. 2019, Article ID 8641492, 19 pages, 2019.

[29] D. Chemla, F. Meunier, and R. Wolfler Calvo, "Bike sharing systems: solving the static rebalancing problem," Discrete Optimization, vol. 10, no. 2, pp. 120-146, 2013.

[30] R. Nair and E. Miller-Hooks, "Fleet management for vehicle sharing operations," Transportation Science, vol. 45, no. 4, pp. 524-540, 2011.

[31] P. Angeloudis, J. Hu, and M. G. H. Bell, "A strategic repositioning algorithm for bicycle-sharing schemes," Transportmetrica A: Transport Science, vol. 10, no. 8, pp. 759-774, 2014.

[32] I. A. Forma, T. Raviv, and M. Tzur, "A 3-step math heuristic for the static repositioning problem in bike-sharing systems," Transportation Research Part B: Methodological, vol. 71, pp. 230-247, 2015.

[33] M. Casazza, A. Ceselli, D. Chemla, F. Meunier, and R. Wolfler Calvo, "The multiple vehicle balancing problem," Networks, vol. 72, no. 3, pp. 337-357, 2018.

[34] S. C. Ho and Y. S. Wai, "A hybrid large neighborhood search for the static multi-vehicle bike-repositioning problem," Transportation Research Part B: Methodological, vol. 95, pp. 340-363, 2017.

[35] T. L. Júnior, "The static bike relocation problem with multiple vehicles and visits," European Journal of Operational Research, vol. 264, pp. 508-523, 2018.

[36] S. Ghosh, P. Varakantham, Y. Adulyasak, and P. Jaillet, "Dynamic repositioning to reduce lost demand in bike sharing systems," Journal of Artificial Intelligence Research, vol. 58, pp. 387-430, 2017.

[37] W. Y. Szeto and C. Shui, "Exact loading and unloading strategies for the static multi-vehicle bike repositioning problem," Transportation Research Part B: Methodological, vol. 109, pp. 176-211, 2018.
[38] A. Pal and Z. Yu, "Free-floating bike sharing: solving real-life large-scale static rebalancing problems," Transportation Research Part C: Emerging Technologies, vol. 80, pp. 92-116, 2017.

[39] Z.-Y. Zhang and X. Zhang, "Shared bikes scheduling under users' travel uncertainty," IEEE Access, vol. 8, pp. 3123-3143, 2020.

[40] Y. Liu, "A static free-floating bike repositioning problem with multiple heterogeneous vehicles, multiple depots, and multiple visits," Transportation Research Part C: Emerging Technologies, vol. 92, pp. 208-242, 2018.

[41] L. Hubert and P. Arabie, "Comparing partitions," Journal of Classification, vol. 2, pp. 193-218, 1985.

[42] B. Djsa and C. Xd, "Spatiotemporal evolution of ridesourcing markets under the new restriction policy: a case study in Shanghai," Transportation Research Part A: Policy and Practice, vol. 130, pp. 227-239, 2019.

[43] Q. Zhou, C. J. Wong, and X. Su, "Machine learning approach to quantity management for long-term sustainable development of dockless public bike: case of shenzhen in China," Journal of Advanced Transportation, vol. 2020, no. 1, Article ID 8847752, 13 pages, 2020. 